\title{
Modelling vibratory roller-soil system dynamics using discrete element method
}

\author{
Jian Wang(1,2), Aimin Sha(3), Liqun $\mathrm{Hu}^{(3)}$ \\ (1) School of Highway, Chang'an University, Xi'an, Shannxi Province, CHINA \\ e-mail: jianwang0712@gmail.com \\ (2) School of Civil and Transportation Engineering, Henan University of Urban Construction, Pingdingshan, Henan, CHINA \\ (3) Key Laboratory for Special Area Highway Engineering of Ministry of Education, Chang'an University, Xi'an, Shaanxi, CHINA
}

\section{SUMMARY}

A simple yet useful implementation of lumped-parameter models using the discrete element method is discussed in this paper. Lumped-parameter models are often used in the modelling of vibratory roller-soil systems owing to their ability to capture the essential features observed during roller-soil vibrations. In order to investigate the system behaviour in the event of a decoupling (loss of contact between the drum and the soil), two conditions pertaining to the transition between the contact and non-contact phases are compared in the light of simulation results. The results demonstrate significant differences in the roller-soil system behaviour based on operating conditions. One particular case provides an unrealistic estimate of the contact force, and thus may lead to an incorrect prediction of the system dynamics. Another solution, however, provides a reasonable estimate of the system dynamics and is recommended herein.

KEY WORDS: lumped-parameter model; discrete element method; vibratory roller-soil system.

\section{INTRODUCTION}

Instrumented vibratory roller compactors are increasingly used in the pavement construction owing to their ability to provide continuous assessment of soil stiffness for the purposes of quality control (QC). The roller-based measurement, however, requires accurate modelling of the roller-soil system. Data collected from the field experiments involving the instrumented roller compactors have revealed a fairly complex nonlinear behaviour of the system, including decoupling [1, 2], drum and frame rocking [1, 3], and chaotic behaviour [1, 4, 5]. In addition, a traveling roller that interacts with the underlying soil heterogeneity and operates over a variety of excitation frequencies and amplitudes leads to a highly transient behaviour [2-4].

Various modelling methodologies were employed in the past to model dynamic roller-soil interactions, such as the well-known lumped-parameter model $[2,6]$, continuum-based finite element (FE) analysis [7-9], and boundary element (BE) analysis [8]. In the lumped-parameter approach, the soil is modelled as a discrete mass-spring-dashpot element, thereby 'lumping' 
together all of the ground into a single object. This approach has been successfully employed to characterise dynamic drum-soil interactions $[2,5,6,10,11]$. However, it cannot be used to model individual layers and curved drum interactions with soil. Such issues have been addressed using FE and $\mathrm{BE}$ analyses techniques [7, 8], wherein the soil is assumed to be linearly elastic, isotropic, and homogeneous, which, however, is not true for most granular materials.

Due to the limitations of the traditional FE analysis in the modelling of granular soil, many researchers seek alternative modelling techniques, especially those particle-based methods, in which discrete particles are used to model the soil and inter-particle forces used to model the soil mechanical behaviour. These include: (i) SPH (Particle Finite Element Method), (ii) MPM (Material Point Method), (iii) PFEM (Particle Finite Element Method) and (iv) DEM (Discrete Element Method).

In the SPH [12], the particles are used as interpolation points for solving the continuum mechanics equations (i.e., Cauchy equation of motion in the case of soils). It is a mesh-free method and the continuum equations are discretised for each particle using a kernel smoothing function. $[13,14]$ used a coupled FEA/SPH model to simulate the rolling rigid and flexible tires on a soft soil. They used a hydrodynamic elastic-plastic material for the soil. Although the SPH model seems promising, the material models used need to be further refined to avoid excessive viscosity or incorrect compressibility.

In the MPM [15], a Cartesian grid is used along with the particles for detecting neighbouring particles, discretising and solving the continuum mechanics equations. In [16], this approach was used to model snow and rigid body interaction.

In the PFEM [17], a polyhedral finite element mesh is generated from the particles using an extended Delaunay tessellation. With that mesh, the continuum mechanics equations can be solved. In [18], the PFEM was used to model sand flow from a silo, angle of repose and granular column collapse. The PFEM may require less soil particles, however, the tessellation step during every time step is computationally intensive.

Unlike the above particle-based methods, which are essentially based upon continuum mechanics formulation and rely on constitutive material models, the DEM accounts for soil mechanical behaviour by modelling inter-particle forces and particle motions directly. The particle force models in DEM are easier to develop compared to their continuum mechanics counterparts and DEM is currently the most mature particle method. DEM was originally invented by Cundall and Strack to solve rock mechanics problems [19]. Since then, it has gained increasing attention in granular materials research due to its ability to represent the discrete and heterogeneous nature of granular material [20-23].

The complexity of the interaction between deformable bodies and granular material drives the need for innovative modelling tools capable of combining DEM and continuum numerical methods [24-26]. In [26], the combined FE-DE method was used to model the interactions between a tire tread and granular terrain in which the DEM is used to describe the dynamics of the granular assembly, and the FEM accurately predicts the deformations and stresses acting within the tire tread.

Owing to its inherent advantages in the modelling of multi-body systems, DEM may be the most promising technique for building roller-soil interaction models. However, in the available literature concerning pavement engineering, DEM simulations for vibratory compaction have been mostly focused on assessing the behaviour of granular materials being compacted [27- 
30]. In these studies, the roller was modelled as a static or dynamic load along with some boundary constraints applied to the simulated granular materials.

Unlike most existing DEM approaches, this paper presents a roller-soil interaction model that is based on a simple lumped-parameter model and developed using a general-purpose DEM software package called the particle flow code (PFC). We first introduced a simple lumpedparameter model in section 2 . Then we discussed the details of modelling vibratory roller-soil interactions via the DEM method in section 3, followed by empirical evaluation of the proposed DEM model in section 4. Finally, we drew a conclusion and discussed the prospective work in section 5 .

\section{THE LUMPED-PARAMETER MODEL}

A typical roller-soil interaction model is depicted in Figure 1a, wherein both the roller and soil are represented by mass-spring-dashpot elements. This lumped-parameter model has been repeatedly proven to give good agreement between the field measurements and simulation [4, $6,10,11]$.

The dynamic behaviour of the model could be described using the following equations:

$$
\begin{aligned}
& m_{2} \ddot{x}_{2}=-m_{2} g+F_{c}+c_{23}\left(\dot{x}_{3}-\dot{x}_{2}\right)+k_{23}\left(x_{3}-x_{2}\right)-F_{e} \\
& m_{3} \ddot{x}_{3}=-m_{3} g-c_{23}\left(\dot{x}_{3}-\dot{x}_{2}\right)-k_{23}\left(x_{3}-x_{2}\right)
\end{aligned}
$$

where $m_{1,2,3}$ and $x_{1,2,3}$ represent mass and vertical displacement of soil, roller drum and frame, respectively. The dashpot $c_{23}$ and spring $k_{23}$ elements account for the interaction force between the roller drum and frame. $F_{e}=F_{\omega} \sin (\omega t)$ is an excitation force exerted on roller drum with constant force amplitude $F_{\omega}$ and angular frequency $\omega$, and is a function of vibration time $t . F_{c}$ stands for the contact force between the roller drum and soil and its sign and magnitude is determined by the contact force law which will be discussed further in the following sections. 


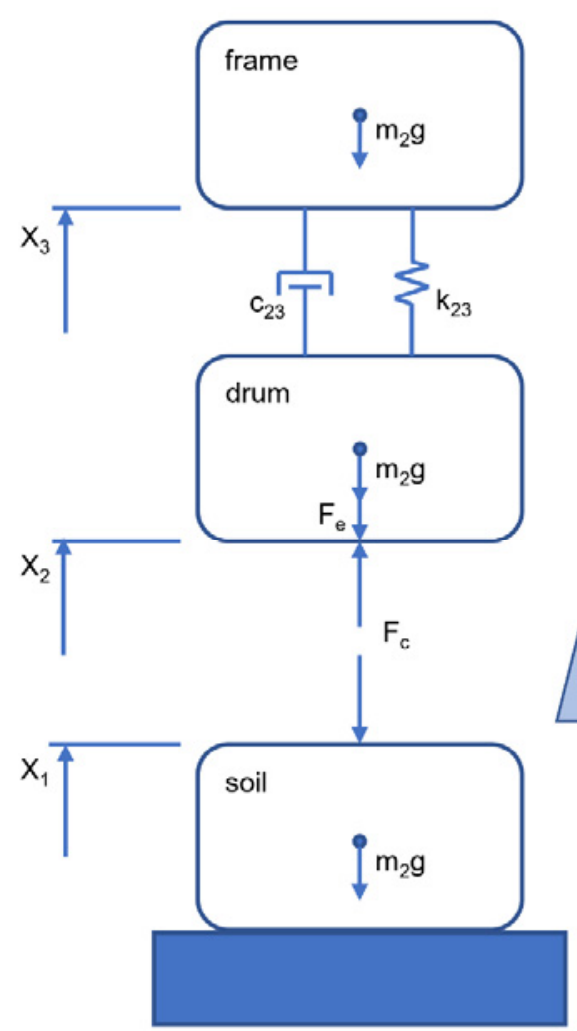

(a)

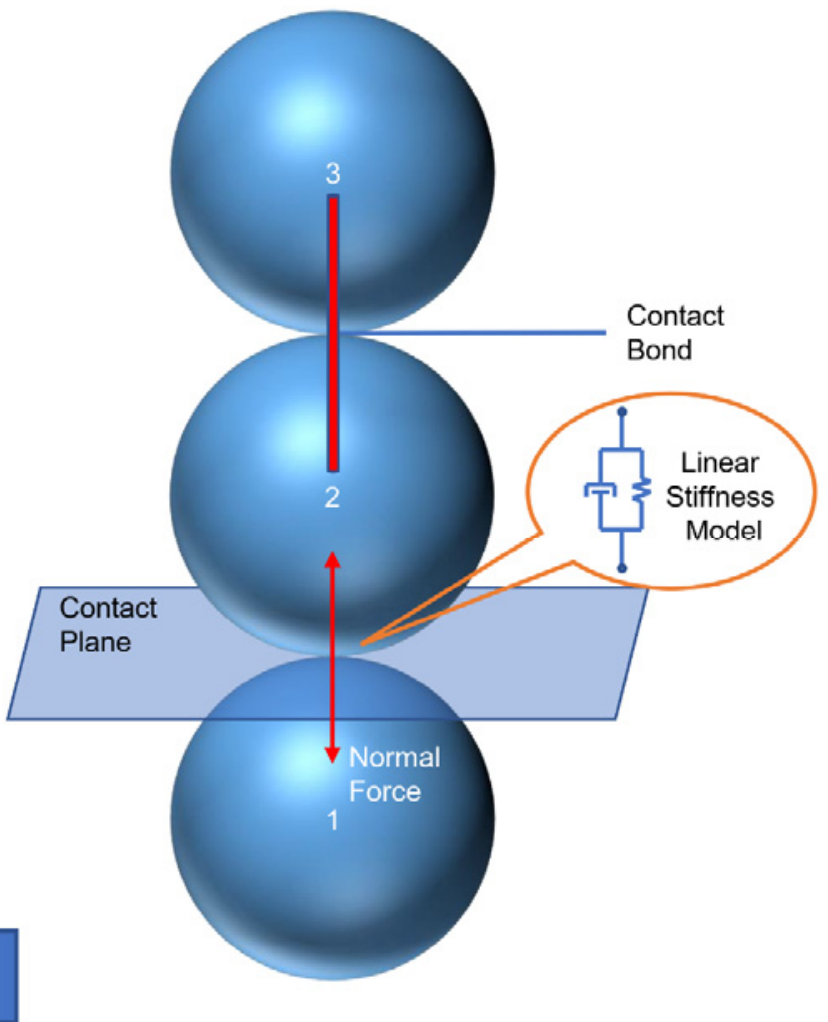

(b)

Fig. 1 Modelling of the roller-soil interaction system with (a) lumped parameter model (b) DEM model

\section{THE PROPOSED DEM MODEL}

\subsection{MODELLING PROCEDURE}

The DEM is an explicit time stepping algorithm applying the basic Newton's second law of motion [19]. In a typical DEM simulation, particles are assumed to be rigid bodies. Interparticle deformations are very small and thus can be simplified as inter-particle overlaps. The time step is small enough that the velocities and accelerations of particles can be treated as constant values during each time step. Then, the inter-particle contact forces and moments are calculated by contact laws. The calculated forces are used to compute and update the position and velocity of the particle by time step integration. This cycle is repeated until the end of the simulation. In this study, the classical general-purpose DEM software called PFC (Particle Flow Code) is employed.

"Mirrored" from the lumped-parameter model in Figure 1a, the concerned roller-soil system was modelled using DEM and is depicted in Figure 1b. The proposed model comprises three balls, each of which represents an integral part of the roller-soil system.

The modelling procedure was performed in the following steps.

1. Three balls were arranged in line with their translational motion inhibited in the horizontal direction. So only the contact forces acting in directions that are normal to 
the contact plane are allowed (Figure 1b). The initial positions of the balls were chosen to ensure that the neighbouring balls remain in contact but do not overlap with each other. The initial velocities of the balls were zero.

2. The balls were then allowed to settle under gravity until the attainment of static equilibrium.

3. Excitation force was applied to the drum (ball 2) and the parameters of interest (e.g., displacement, velocity, and acceleration of the balls) were tracked. The recorded data at a steady state were analysed in this study.

A linear stiffness contact model with viscous damping (hereinafter: linear contact model) was used for ball-ball contacts as shown in Figure 1b. This is similar to the parallel spring-dashpot employed in a lumped-parameter system as shown in Figure 1a. Figure $1 \mathrm{~b}$ also shows two types of contacts in the model: The contact between the drum (ball 2) and frame (ball 3) was of the bonded type (i.e., it can resist the tensile force), while no bonding existed between the soil (ball 1) and drum (ball 2). The bond strength between the drum and frame was maintained large enough to avoid breaking. Although drum-frame interactions form an essential part in the model, our main focus in the case at hand was on the roller-soil interactions. The contactforce law is given by:

$$
F_{c}= \begin{cases}c\left(\dot{x}_{1}-\dot{x}_{2}\right)+k\left(x_{1}-x_{2}\right), & \text { during contact phase } \\ 0 . & \text { during non-contact phase }\end{cases}
$$

where $c$ represents the damping coefficient; $k$ is the contact stiffness; $\left(x_{1}-x_{2}\right)$ is the distance between the contacting surfaces, while $\left(x_{1}-x_{2}\right)>0$ is overlapping (Figure 2); and $\left(\dot{x}_{1}-\dot{x}_{2}\right)$ is the relative velocity between the two contacting balls, while $\left(\dot{x}_{1}-\dot{x}_{2}\right)>0$ indicates the increasing overlap (Figure 2).

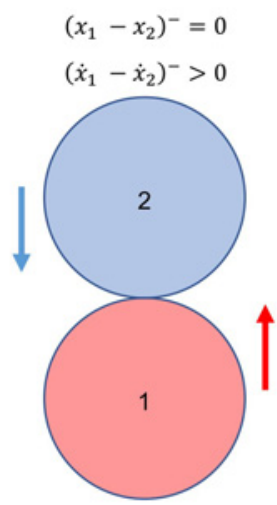

(a)

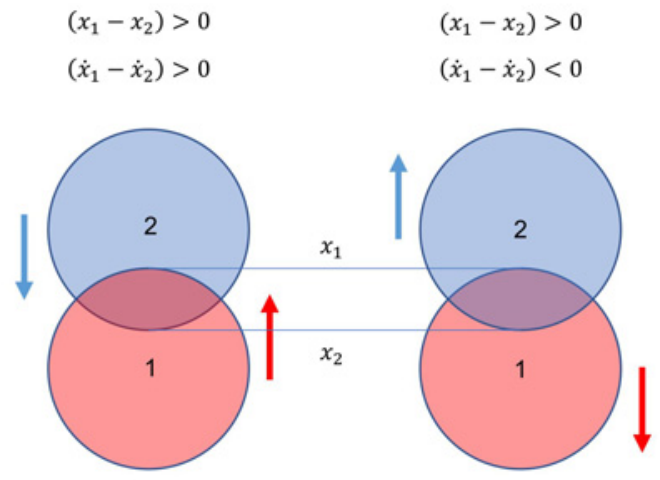

(b)

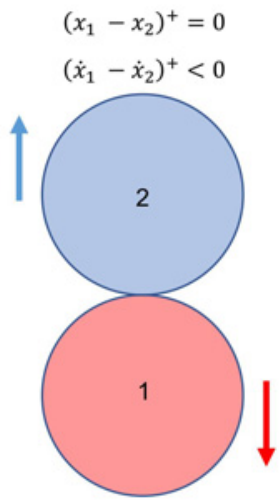

(d)

Fig. 2 Contact evolution in a DEM model when contact overlap is (a) starting, (b) increasing, (c) decreasing, and (d) finishing

Besides Eq. (1) and (2), the related conditions for the transition from the contact phase to noncontact phase and vice versa should also be defined in order to fully describe the system dynamics and possible decoupling of the soil and drum. In the following sections, we will discuss in detail two transition conditions (denoted as A and B) in DEM, along with their advantages and limitations. For simplicity, we used a superscripted "minus" sign to denote the 
instance when the contact is made and a superscripted "plus" sign to denote when the contact is lost (Figure 2).

\subsection{TRANSITION CONDITIONS}

\subsubsection{TRANSITION CONDITION A}

Under this condition, the creation or loss of contact only happens when the gap/overlap between the balls becomes zero. For the model depicted in Figure 1, it's applies as follows:

$$
x_{1}-x_{2}=0
$$

However, the contact force has a non-zero value owing to viscous damping. From Eq. (2), by setting $\left(x_{1}-x_{2}\right)^{-}=0$, the net force that exists when the contact is made may be obtained by using:

$$
F_{c}^{A-}=c\left(\dot{x}_{1}-\dot{x}_{2}\right)^{-}
$$

where $\left(\dot{x}_{1}-\dot{x}_{2}\right)^{-}>0$ hence the initial contact force is greater than zero (compression). In other words, at the instance when the contact is made, the magnitude of the contact force increases from zero to a non-zero value instantly, hence a discontinuity in the force is to be observed.

Similarly, when the contact is lost, the net force is given by:

$$
F_{c}^{A+}=c\left(\dot{x}_{1}-\dot{x}_{2}\right)^{+}
$$

where $\left(\dot{x}_{1}-\dot{x}_{2}\right)^{+}<0$, thereby indicating the action of the tensile force at the instance when the contact is lost. This may lead to unrealistic behaviour, since in most cases, the adhesive force between the soil and drum is very weak and thus negligible. Also, since the magnitude of the contact force drops to zero instantly, a discontinuity in force (as a function of time) is to be expected.

\subsubsection{TRANSITION CONDITION B}

At the beginning of the contact, transition condition A and B are basically identical. The difference lies in the different definitions of when the contact ends. At the end of the contact, condition B can be expressed by:

$$
F_{c}^{B+}=0
$$

which means the total contact force at the end of the contact is zero. So, no unrealistic adhesive (tensile) forces originate from the transition condition B. The contact force changes continuously to zero at the end of the contact. Substituting Eq. (2) into Eq. (6) the relative displacement (contact overlap) between ball 1 and ball 2 at the end of the contact is given by:

$$
\left(x_{1}-x_{2}\right)^{+}=-\frac{c\left(\dot{x}_{1}-\dot{x}_{2}\right)^{+}}{k}
$$

Combining Eq. (3) and Eq. (6), we obtain: 


$$
\left(x_{1}-x_{2}\right)^{-}=0 \wedge F_{c}^{B+}=0
$$

Similarly, from Eq. (7), it derives:

$$
F_{c}^{B-}>0 \wedge\left(x_{1}-x_{2}\right)^{+}>0
$$

where $F_{c}^{B-}>0$ implies the existence of the compressive force when the contact is made, and $\left(x_{1}-x_{2}\right)^{+}>0$ implies an overlapping of balls 1 and 2 when the contact is lost. In other words, although the two balls are overlapping, the net contact force is zero.

The principal contact behaviours for a single impact corresponding to transition conditions A and $B$ are summarised in Figure 3, whereas the dotted line represents the difference between the two.

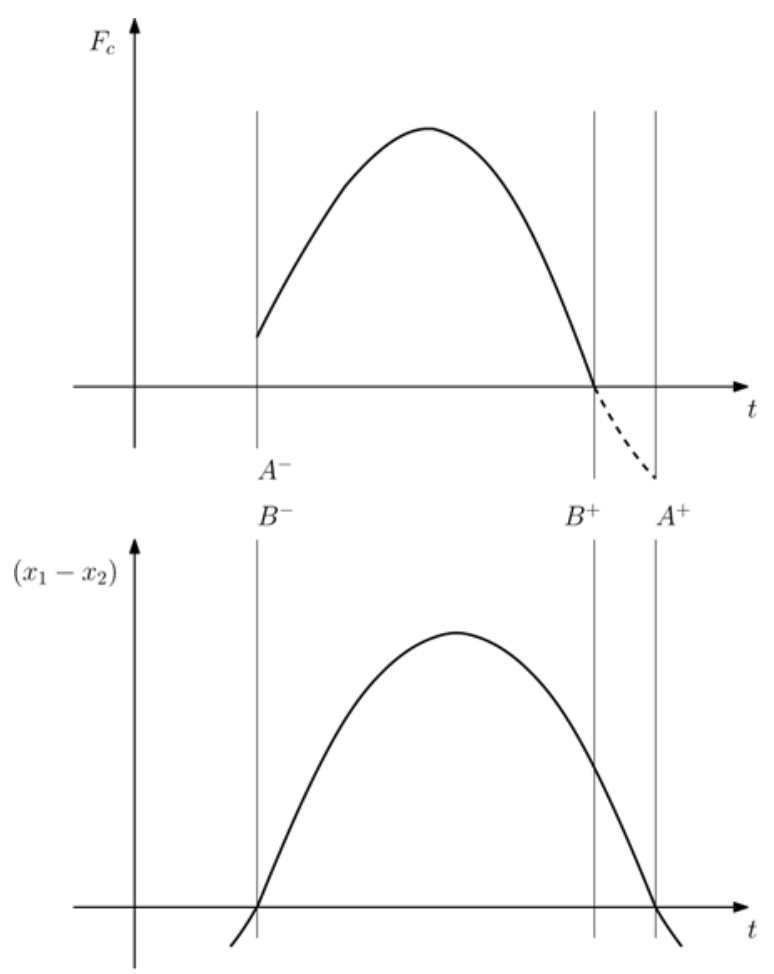

Fig. 3 Contact behaviour corresponding to transition conditions $A$ and $B$

\subsection{MODELLING PARAMETERS}

Usually, it is necessary to run some simulations using the proposed model and compare the results with the field data to validate the model. However, since the validity of using the lumped parameter model to model roller-soil dynamics has been proven by many studies, we can safely assert that the proposed DEM model has the same level of validity as the lumped parameter model if we could demonstrate their equivalency by running simulations using the same parameter sets. Nevertheless, since only a small subset of parameter combinations can be feasibly explored, the simulation results we present here are aimed to serve only as examples of the various issues that need to be considered, especially those related to contact transitions. The used parameter combinations are summarised in Table 1. 
Table 1 Combination of modelling-parameter values used in simulations.

\begin{tabular}{ccccc}
\hline Parameters & $\boldsymbol{I}$ & $\boldsymbol{I I}$ & $\boldsymbol{I I I}$ & $\boldsymbol{I V}$ \\
\hline$k(\mathrm{MN} / \mathrm{m})$ & 5 & 100 & 100 & 100 \\
$D$ & 0.5 & 0.5 & 0.5 & 2.0 \\
$c(\mathrm{MNs} / \mathrm{m})$ & 0.1 & 0.447 & 0.447 & 1.79 \\
$\omega(\mathrm{rad} / \mathrm{s})$ & $40 \pi$ & $40 \pi$ & $80 \pi$ & $80 \pi$ \\
\hline
\end{tabular}

In Table 1, the damping coefficient $c$ of the drum-soil contact depends on the drum mass $m_{2}$, the damping ratio $D$ and the contact stiffness $k$ :

$$
c=2 D \sqrt{m_{2} k}
$$

In addition to those listed in Table 1, the common parameters were maintained constant in four simulations assuming the following values: $m_{2}=2 \times 10^{3} \mathrm{~kg}, m_{3}=1 \times 10^{3} \mathrm{~kg}$, $k_{23}=1 \frac{M N}{m}, c_{23}=1 \times 10^{4} \frac{\mathrm{Ns}}{\mathrm{m}}, g=9.81 \frac{\mathrm{m}}{\mathrm{s}^{2}}$ and $F_{\omega}=58.8 \times 10^{3} \mathrm{~N}$. We have run simulations for the abovementioned parameter combinations under both transition conditions A and B. The results will be compared and discussed in the following section.

\section{MODELLING RESULTS}

A series of simulations was performed under both transition conditions A and B. In this section, we first analysed in detail the results from the four chosen cases under different transition conditions. Then, we compared the modelling results of the proposed DEM with those solved by other numerical tools. Finally, we concluded with a discussion of future research prospects of extending the proposed model to a multi-scale model.

\subsection{ANALYSIS}

\subsubsection{CASE I}

Figure 4 shows the recorded acceleration of the roller drum (ball 2) after steady state was reached as a function of time. From Figure 4, it is clear that the drum acceleration under conditions $\mathrm{A}$ and $\mathrm{B}$ is identical, indicating that no transition occurred. This is due to the excitation force and the resulting drum-soil contact force which is not large enough to overcome the gravitational force acting on roller. Hence, the roller stayed on the ground and no impact occurred. In other words, no drum-soil decoupling happened. 


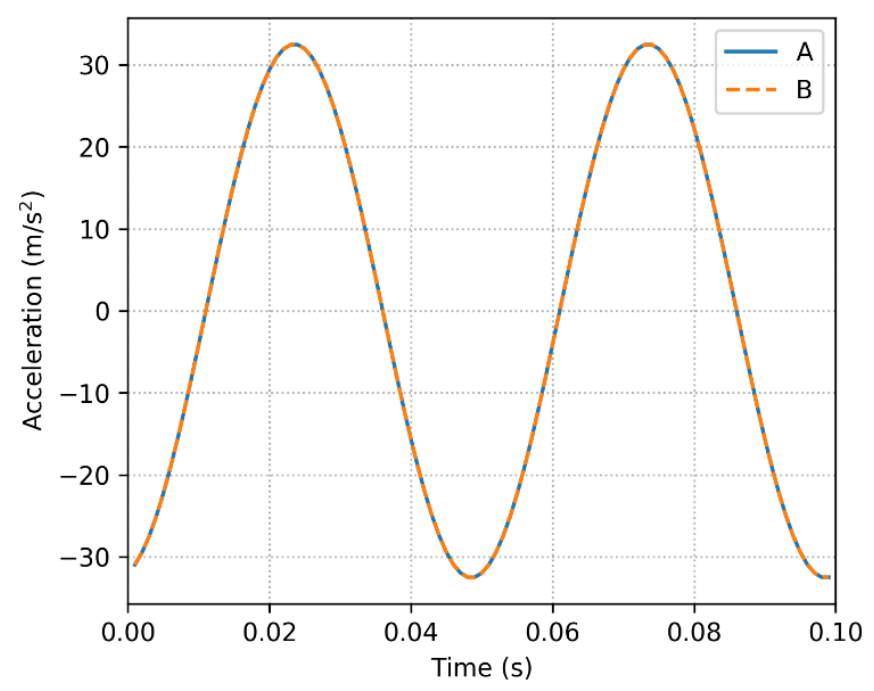

Fig. 4 Drum acceleration at a steady state (Case I)

\subsubsection{CASE II}

In this case, the contact stiffness $\mathrm{k}$ was raised to a value 20 times larger compared to Case I. Also, according to Eq. (10), the damping coefficient $c$ increases with $k$. However, the increased damping force was not large enough to prevent the drum from losing contact with the soil. This was proven by the resulting acceleration of the drum at a steady state depicted in Figure 5. The figure shows considerably different system dynamics for transition conditions A and B, thereby indicating the presence of decoupling of the drum and soil after the system attains the steady state. In addition, the previously discussed discontinuity due to the presence of unrealistic adhesive forces in the transition condition A can be clearly observed in Figure 5.

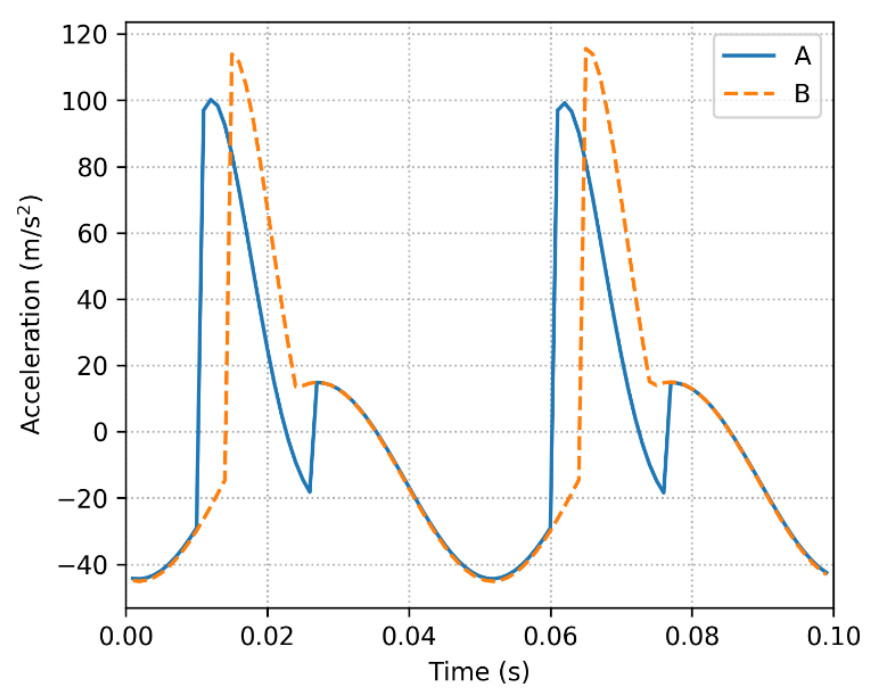

Fig. 5 Drum acceleration at a steady state (Case II) 


\subsubsection{CASE III}

In comparison to Case II, the only difference in Case III was that the angular frequency is doubled. Hence, the observed cycles within the same amount of time have doubled (Figure 6). The overall curve shapes are similar to those in Figure 5 except that the maximum drum acceleration is approximately half of that obtained for Case II. This is because the time for developing the soil deformation has been significantly shortened, resulting in the reduction in the maximum contact force and ultimately, the maximum drum acceleration.

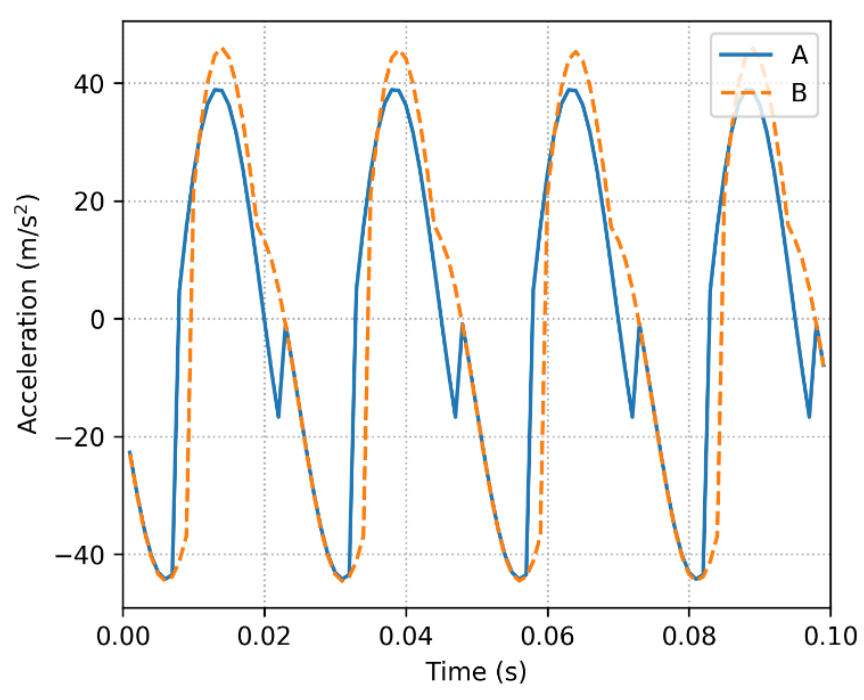

Fig. 6 Drum acceleration at steady state (Case II)

\subsubsection{CASE IV}

With this set of parameters, significant differences between transition conditions A and B were once again evident (Figure 7). For the transition condition A, since the viscous damping was 4 times larger as compared to Case I (damping ratio D raised from 0.5 to 2.0), the resulting tensile force was large enough to prevent the loss of contact. This is apparent in Figure 7, where the curve corresponding to the transition condition A remains in the sinusoidal shape. In contrast, the shape of the dashed line in Figure 7 indicates that the decoupling occurs. This is because no tensile force exists for the transition condition B. Also, due to large damping, the compressive damping force at the beginning of the contact for the transition condition $\mathrm{B}$ is also non-negligible, resulting in a discontinuity in the drum acceleration for each cycle, as shown in Figure 7. 


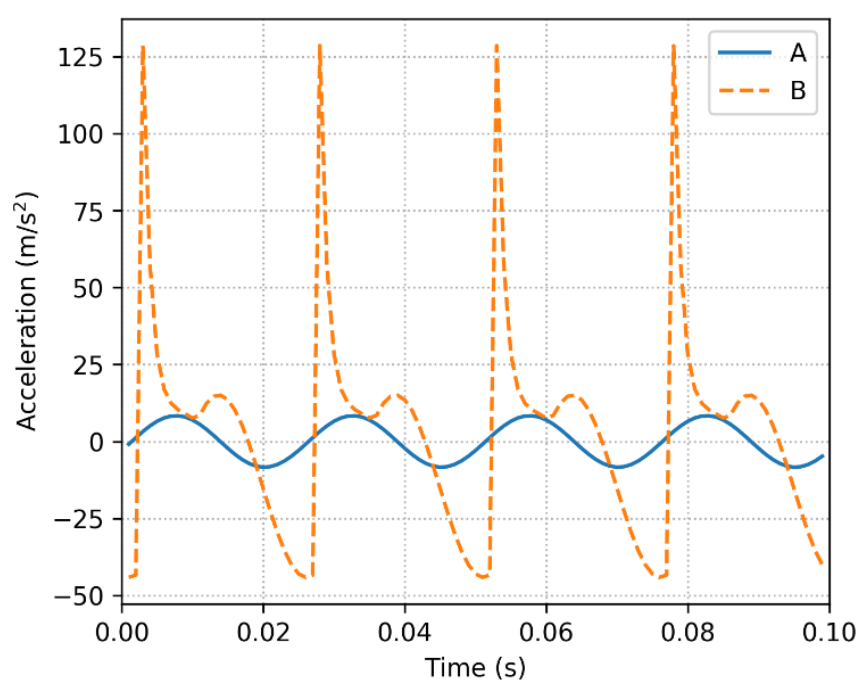

Fig. 7 Drum acceleration at a steady state (Case II)

As obvious from the above simulation results, different transition conditions lead to largely different predictions of the system dynamics. Transition condition B is seen to provide a rather realistic prediction for the roller-soil system dynamics, whereas condition A leads to an unrealistic estimation of the system dynamics. The authors, therefore, recommend the use of condition B in the modelling to the roller-soil systems intended for practical use.

\subsection{COMPARISON TO THE RESULTS FROM DIRECT SOLVING}
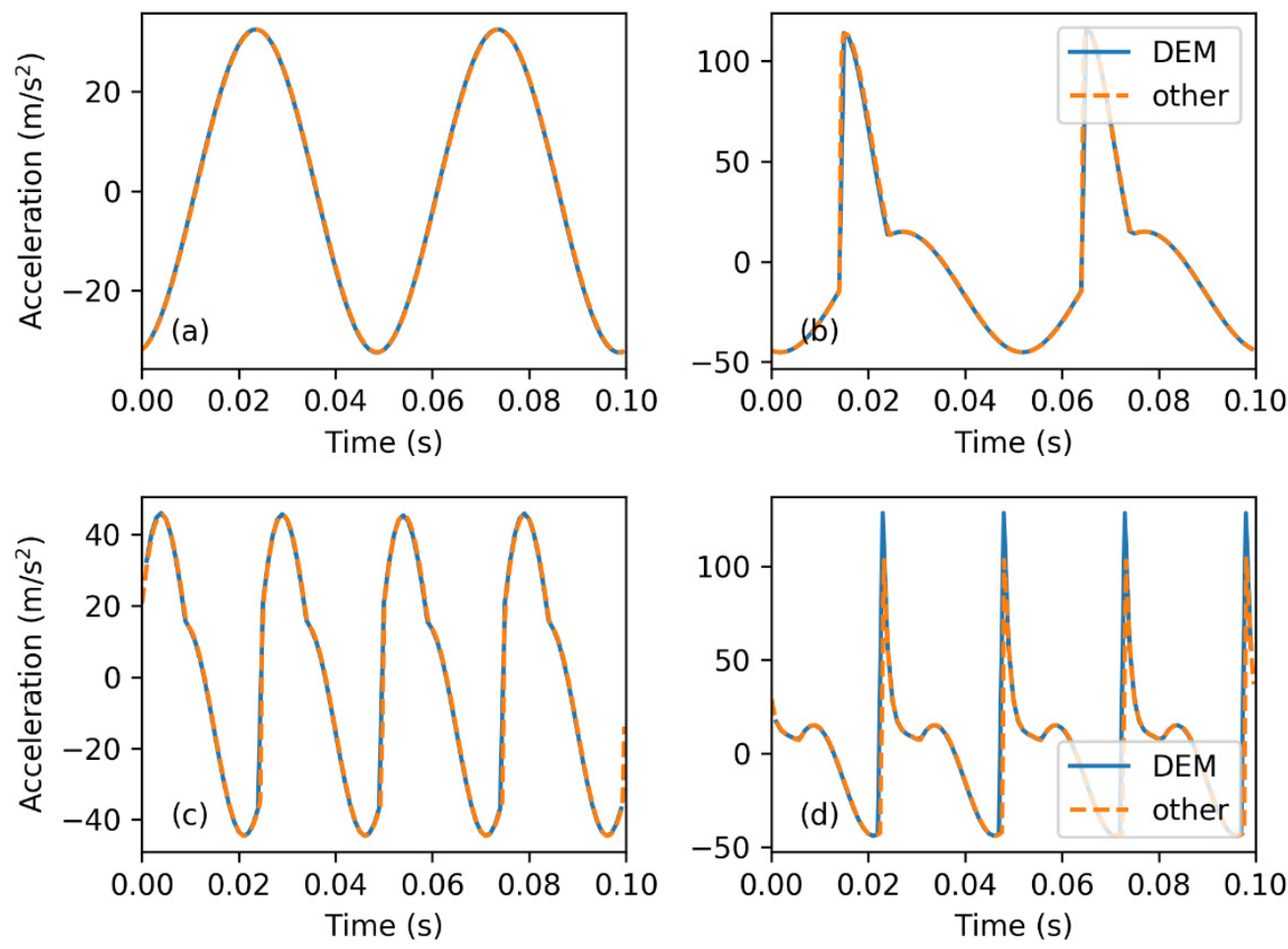

Fig. 8 The simulation results from the proposed DEM model and other techniques of (a) Case I, (b) Case II, (c) Case III, (d) Case IV 
The results from the proposed DEM model were also compared to those from other techniques. The lumped parameter model (i.e., Eq. (1) and Eq. (2)) was solved directly with other software packages such as MATLAB. Figure 8 depicts the results from both approaches corresponding to the transition condition B. It is evident that the results from the proposed DEM model are basically identical to those solved directly. Therefore, the proposed DEM modelling technique could be used to model the roller-soil system interactions and the concerned dynamic response of the system.

\subsection{EXTENDING TO A MULTI-SCALE MODEL}

The above proposed DEM model is a simplified, one-dimensional model. Nevertheless, the model has the potential to be extended to a multi-scale model by simply replacing the ball representing the soil (ball 1 in Figure 1) with a granular assembly. Figure 9 shows such a model in which the soil is represented by a granular assembly consisting of 2500 balls. The "soil" particles were generated randomly in a box and allowed to settle under gravity. Then the two "roller" balls were created on top of the soil (Figure 9). The creation of roller balls should follow the same protocol described in section 3. Also, the translational motion in horizontal direction and the rotational motion of the "roller" should be inhibited to stop the "roller" from tipping over.

The macroscopic behaviour of the soil in the multi-scale model is influenced by many microscopic parameters (e.g. particle size distribution, packing characteristics, inter-particle friction, etc.) and vibration parameters. This provides a good connection between the microscopic properties and the macroscopic behaviour. Note that although the model shown in Figure 9 is a two-dimensional DEM model, the same technique can be easily generalised to a more sophisticated, three-dimensional model. Moreover, a more realistic drum shape can be formed by adopting the clumped ball technique.

In general, the multi-scale DEM model has the potential to model both the deformation of the granular soil and the roller dynamics simultaneously while keeping the model simple. However, even though the model is simple in nature, the resulting responses may be rather complex. It is critical to calibrate the model before using it for prediction of the field data. The calibration process is out of the scope of the present study and is deferred to future work. 


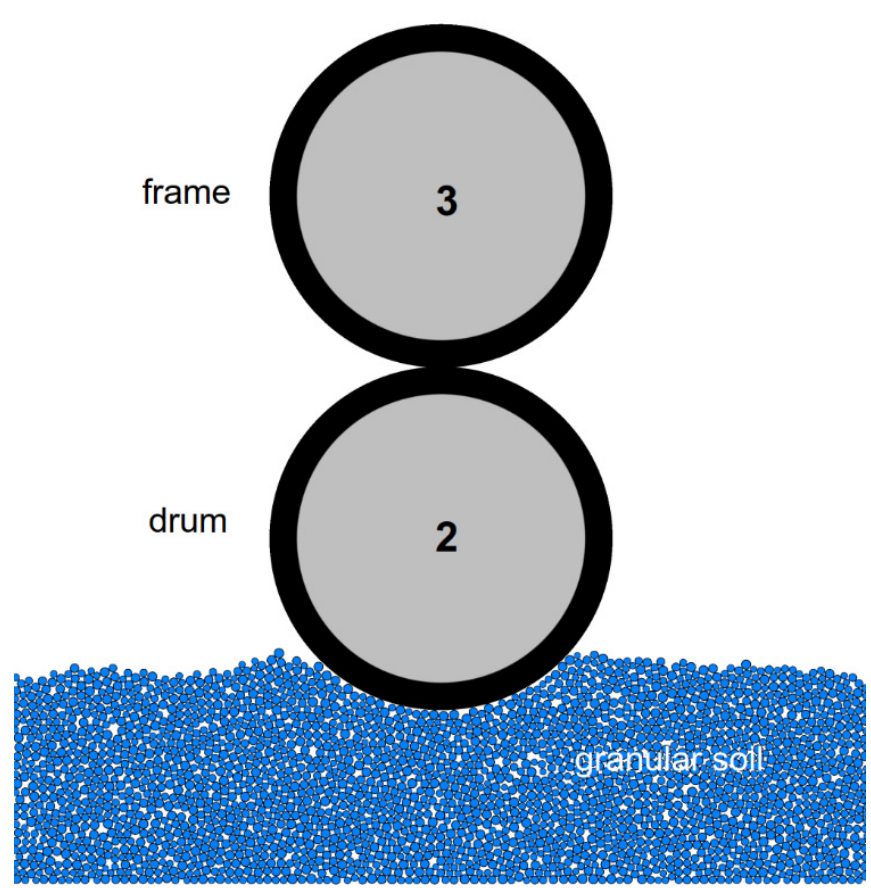

Fig. 9 Vibratory roller and granular soil modelled with DEM

\section{CONCLUSION}

This study presents the development of the DEM method to model the roller-soil interactions based on the lumped-parameter system. By performing simulations under various parameter combinations and analysing the simulated roller-soil dynamics, the following conclusions have been drawn.

1. A lumped-parameter model can be seamlessly replaced by an equivalent DEM model. The DEM modelling procedure is simple and more intuitive compared to the lumpedparameter that requires direct solving.

2. Transition conditions play an important role in the simulated system dynamics. It is critical to select a transition condition that does not produce unrealistic forces. For the model proposed in this paper, the transition condition B is recommended for the best results.

3. The proposed DEM model is highly extensible. By replacing the ball 1 (representing soil in Figure 1) in the model, we can build a multi-scale model in which both the granular soil and roller dynamics can be simulated more realistically compared to other modelling techniques. The proposed DEM model can be easily extended to a multi-scale model by replacing the ball representing soil with a granular assembly.

The proposed DEM model presented in this paper is a preliminary, one-dimensional model. Future work will focus on the validation and calibration of the multi-scale vibratory roller-soil model using field data. 


\section{REFERENCES}

[1] J.-L. Briaud and J. Seo, Intelligent compaction: overview and research needs, Texas A\&M University, 2003.

[2] P.J. van Susante and M.A. Mooney, Capturing nonlinear vibratory roller compactor behavior through lumped parameter modeling, Journal of Engineering Mechanics, Vol. 134, No. 8, pp. 684-693, 2008.

https://doi.org/10.1061/(ASCE)0733-9399(2008)134:8(684)

[3] N.W. Facas, P.J. van Susante and M.A. Mooney, Influence of rocking motion on vibratory roller-based measurement of soil stiffness, Journal of Engineering Mechanics, Vol. 136, No. 7, pp. 898-905, 2009.

https://doi.org/10.1061/(ASCE)EM.1943-7889.0000132

[4] M.A. Mooney and R.V. Rinehart, Field monitoring of roller vibration during compaction of subgrade soil, Journal of Geotechnical and Geoenvironmental Engineering, Vol. 133, No. 3, pp. 257-265, 2007. https://doi.org/10.1061/(ASCE)1090-0241(2007)133:3(257)

[5] R. Anderegg and K. Kaufmann, Intelligent compaction with vibratory rollers: Feedback control systems in automatic compaction and compaction control, Transportation Research Record: Journal of the Transportation Research Board, No. 1868, pp. 124-134, 2004. https://doi.org/10.3141/1868-13

[6] T.-S. Yoo and E.T. Selig, Dynamics of vibratory roller compaction, Journal of Geotechnical and Geoenvironmental Engineering, Vol. 105, No. ASCE 14907 Proc Paper, p. 1979.

[7] O.M. Musimbi, Experimental and numerical investigation of vibratory drum interacting with layered elastic media, 2011.

[8] M.A. Mooney and N.W. Facas, Extraction of layer properties from intelligent compaction data, 2013.

[9] C. Capraru, J. Pistrol, S. Villwock, W. Völkel, F. Kopf and D. Adam, Numerical simulation of soil compaction with oscillatory rollers, In: XV Danube European Conference on Geotechnical Engineering, Vienna, pp. 283-288, 2014.

[10] D. Pietzsch and W. Poppy, Simulation of soil compaction with vibratory rollers, Journal of Terramechanics, Vol. 29, No. 6, pp. 585-597, 1992.

https://doi.org/10.1016/0022-4898(92)90038-L

[11] S. Natsiavas, Dynamics of multiple-degree-of-freedom oscillators with colliding components, Journal of Sound and Vibration, Vol. 165, No. 3, pp. 439-453, 1993.

https://doi.org/10.1006/jsvi.1993.1269

[12] J.J. Monaghan, Smoothed particle hydrodynamics, Reports on Progress in Physics, Vol. 68, No. 8, p. 1703, 2005.

https://doi.org/10.1088/0034-4885/68/8/R01

[13] R. Lescoe, M. El-Gindy, K. Koudela, F. Öijer, M. Trivedi and I. Johansson, Tire-soil modeling using finite element analysis and smooth particle hydrodynamics techniques, In: ASME 2010 International Design Engineering Technical Conferences and Computers 
and Information in Engineering Conference, pp. 3-18, American Society of Mechanical Engineers, 2010. https://doi.org/10.1115/DETC2010-28002

[14] R.S. Dhillon, R. Ali, M. El-Gindy, D. Philipps, F. Oijer and I. Johansson, Development of truck tire-soil interaction model using FEA and SPH, SAE Technical Paper, 2013.

[15] D. Sulsky, S.-J. Zhou and H.L. Schreyer, Application of a particle-in-cell method to solid mechanics, Computer Physics Communications, Vol. 87, No. 1-2, pp. 236-252, 1995. https://doi.org/10.1016/0010-4655(94)00170-7

[16] A. Stomakhin, C. Schroeder, L. Chai, J. Teran and A. Selle, A material point method for snow simulation, ACM Transactions on Graphics (TOG), Vol. 32, No. 4, p. 102, 2013.

https://doi.org/10.1145/2461912.2461948

[17] S.R. Idelsohn, E. O-ate and F.D. Pin, The particle finite element method: a powerful tool to solve incompressible flows with free-surfaces and breaking waves, International Journal for Numerical Methods in Engineering, Vol. 61, No. 7, pp. 964-989, 2004.

https://doi.org/10.1002/nme.1096

[18] E. O-ate, M.A. Celigueta, S.R. Idelsohn, F. Salazar and B. Suárez, Possibilities of the particle finite element method for fluid-soil-structure interaction problems, Computational Mechanics, Vol. 48, No. 3, p. 307, 2011.

https://doi.org/10.1007/s00466-011-0617-2

[19] P.A. Cundall and O.D.L. Strack, A discrete numerical model for granular assemblies, Géotechnique, Vol. 29, No. 1, pp. 47-65, 1979.

https://doi.org/10.1680/geot.1979.29.1.47

[20] H.J. Herrmann and S. Luding, Modeling granular media on the computer, Continuum Mechanics and Thermodynamics, Vol. 10, No. 4, pp. 189-231, 1998.

https://doi.org/10.1007/s001610050089

[21] Ricardo Dobry and Tang-Tat Ng, Discrete modelling of stress-strain behaviour of granular media at small and large strains, Engineering Computations, Vol. 9, No. 2, pp. 129-143, 1992. https://doi.org/10.1108/eb023853

[22] F. Calvetti, G. Viggiani and C. Tamagnini, A numerical investigation of the incremental behavior of granular soils, RIVISTA ITALIANA DI GEOTECNICA, pp. 11-29, 2003.

[23] C. O'Sullivan, Particulate discrete element modelling: a geomechanics perspective, Taylor \& Francis, 2011.

[24] H. Chen, Y.X. Zhang, M. Zang and P.J. Hazell, An accurate and robust contact detection algorithm for particle-solid interaction in combined finite-discrete element analysis, International Journal for Numerical Methods in Engineering, Vol. 103, pp. 598-624, 2015. https://doi.org/10.1002/nme.4913

[25] C.T. Senseney, Z. Duan, B. Zhang and R.A. Regueiro, Combined spheropolyhedral discrete element (DE)-finite element (FE) computational modeling of vertical plate loading on cohesionless soil, Acta Geotechnica, Vol. 12, No. 3, pp. 593-603, 2017.

https://doi.org/10.1007/s11440-016-0519-8 
[26] M. Michael, F. Vogel and B. Peters, DEM-FEM coupling simulations of the interactions between a tire tread and granular terrain, Computer Methods in Applied Mechanics and Engineering, Vol. 289, pp. 227-248, 2015.

https://doi.org/10.1016/j.cma.2015.02.014

[27] L. Wang, B. Zhang, D. Wang and Z. Yue, Fundamental mechanics of asphalt compaction through FEM and DEM modeling, In: Analysis of Asphalt Pavement Materials and Systems: Engineering Methods, pp. 45-63, 2007.

https://doi.org/10.1061/40924(308)5

[28] B. Huang, M. Zhang and J. Yong, Numerical simulation of irregular particle microresponse under vibrating compressive load by DEM, Journal of Highway and Transportation Research and Development, Vol. 26, No. 7, pp. 7-12, 2009.

[29] J. Chen, Discrete element method (DEM) analyses for hot-mix asphalt (HMA) mixture compaction, 2011.

[30] J. Chen, B. Huang, X. Shu and C. Hu, DEM simulation of laboratory compaction of asphalt mixtures using an open source code, Journal of Materials in Civil Engineering, Vol. 27, No. 3, p. 04014130, 2014. https://doi.org/10.1061/(ASCE)MT.1943-5533.0001069 